\title{
Ultimate Limits for Multiple Quantum Channel Discrimination
}

\author{
Quntao Zhuang $\oplus^{1,2, *}$ and Stefano Pirandola $\oplus^{3}$ \\ ${ }^{1}$ Department of Electrical and Computer Engineering, University of Arizona, Tucson, Arizona 85721, USA \\ ${ }^{2}$ James C. Wyant College of Optical Sciences, University of Arizona, Tucson, Arizona 85721, USA \\ ${ }^{3}$ Department of Computer Science, University of York, York YO10 5GH, United Kingdom
}

(Received 8 March 2020; accepted 28 July 2020; published 21 August 2020)

\begin{abstract}
Quantum hypothesis testing is a central task in the entire field of quantum information theory. Understanding its ultimate limits will give insight into a wide range of quantum protocols and applications, from sensing to communication. Although the limits of hypothesis testing between quantum states have been completely clarified by the pioneering works of Helstrom in the 1970s, the more difficult problem of hypothesis testing with quantum channels, i.e., channel discrimination, is less understood. This is mainly due to the complications coming from the use of input entanglement and the possibility of employing adaptive strategies. In this Letter, we establish a lower limit for the ultimate error probability affecting the discrimination of an arbitrary number of quantum channels. We also show that this lower bound is achievable when the channels have certain symmetries. As an example, we apply our results to the problem of channel position finding, where the goal is to identify the location of a target channel among multiple background channels. In this general setting, we find that the use of entanglement offers a great advantage over strategies without entanglement, with nontrivial implications for data readout, target detection, and quantum spectroscopy.
\end{abstract}

DOI: 10.1103/PhysRevLett.125.080505

Hypothesis testing is a fundamental method of statistical inference which plays a central role in both classical and quantum information theory. Since the seminal works by Helstrom [1], quantum hypothesis testing [1-4] has been greatly advanced for the binary case, namely for the statistical discrimination between two quantum states or two quantum channels. Quantum channel discrimination (QCD) [5-9] aims at discriminating between different physical processes, modeled as quantum channels and arbitrarily chosen from some known ensemble. Various protocols have demonstrated the advantages of using entanglement in binary $\mathrm{QCD}$, for example quantum illumination [10-14] and quantum reading [15]. It is also known that all resources in any convex resource theory [16] are useful in binary problems of QCD.

While it is clear that entanglement may give an advantage in some scenarios, the ultimate limit of QCD is far from being understood. The first difficulty results from the fact that solving this limit requires a double optimization, where both input states and output measurements need to be optimized. The second complication comes from the possibility of adaptive strategies, which

Published by the American Physical Society under the terms of the Creative Commons Attribution 4.0 International license. Further distribution of this work must maintain attribution to the author(s) and the published article's title, journal citation, and DOI. may strictly outperform nonadaptive ones [17]. So far only special cases have been considered. For unitaries and certain channels, a finite number of probings allow perfect discrimination [18-20]. For binary discrimination of channels with equal priors, the ultimate adaptive performance can also be found or bounded [21,22].

In this Letter, we are finally able to address the most general scenario. We establish the ultimate limits for the adaptive discrimination of an arbitrary number of finitedimensional quantum channels. More precisely, we provide a general bound to the optimal error probability affecting this general multiary discrimination problem, and we also show relevant cases where this bound is achievable. In fact, for a special class of channels with the property of joint teleportation covariance (telecovariance) $[9,22]$, our bound is tight and achieved nonadaptively by using maximally entangled inputs. Furthermore, when the ensemble of channels possesses the geometric uniform symmetry (GUS) [23], our formulas can be greatly simplified.

As an application, we study the ultimate minimum error probability for the problem of channel position finding (CPF), where the position of a target channel has to be identified among an array of $m$ cells, with the remaining $m-1$ cells containing copies of a background channel. This basic problem has implications for various tasks of quantum sensing as discussed in Ref. [24]. It is here studied considering ensembles of quantum erasure channels (QECs), quantum depolarizing channels (QDCs) and qubit amplitude damping channels (QADCs). In particular, for 
QDCs, we show that the use of input entanglement strictly outperforms nonentangled strategies.

Preliminaries.-Before addressing QCD, let us summarize the case of state discrimination. The minimum "Helstrom" error probability affecting the discrimination of $m$ states $\left\{\rho_{n}\right\}_{n=0}^{m-1}$ with priors $\left\{p_{n}\right\}_{n=0}^{m-1}$ is given by

$$
P_{H}\left(\left\{\rho_{n}, p_{n}\right\}\right)=1-\max _{\sum_{n} \Pi_{n}=I} \sum_{n} p_{n} \operatorname{Tr}\left(\rho_{n} \Pi_{n}\right),
$$

where the positive-valued operator measure (POVM) element $\Pi_{n}$ corresponds to the hypothesis that the state is $\rho_{n}$. In the binary case with equal priors, it reduces to [1] $P_{H}=\left(1-\left\|\rho_{1}-\rho_{2}\right\| / 2\right) / 2$, where $\|A\|=\operatorname{tr} \sqrt{A^{\dagger} A}$ is the one-norm. Since evaluating $P_{H}$ is often challenging, we will resort to various bounds [25-33]. To proceed with our study of QCD, we give a continuity bound for $P_{H}$ as stated in the following lemma (proof in [33]).

Lemma 1.-Consider a set of states $\left\{\rho_{n}^{\prime}\right\}_{n=0}^{m-1}$ close to $\left\{\rho_{n}\right\}_{n=0}^{m-1}$ in the sense that $\left\|\rho_{n}-\rho_{n}^{\prime}\right\| \leq \delta_{n}$ for $0 \leq n \leq m-1$. We lower bound the Helstrom limit as

$$
P_{H}\left(\left\{\rho_{n}^{\prime}, p_{n}\right\}\right) \geq P_{H}\left(\left\{\rho_{n}, p_{n}\right\}\right)-\frac{1}{2} \sum p_{n} \delta_{n} .
$$

Adaptive protocols, simulation, and stretching.-With the continuity bound in hand, we now introduce the most general protocol for QCD and its reduction to state discrimination. A general $u$-round adaptive protocol for multiple channel discrimination is depicted in Fig. 1(a). The protocol is allowed to access an unknown $d$-dimensional channel $\mathcal{E}$ for $u$ times, where the unknown channel $\mathcal{E}$ is fixed but chosen from the ensemble $\left\{\mathcal{E}_{n}, p_{n}\right\}_{n=0}^{m-1}$. The unlimited entanglement between all systems involved allows one to push all measurements to the final output $\rho_{\mathcal{E}, u}$. In each round, a subsystem $S_{k}, 1 \leq k \leq u$, is sent through the channel $\mathcal{E}$ and the output $S_{k}^{\prime}$ is collected. Our goal is to lower bound the ultimate error probability $P_{u}$ of the above protocol.
To simplify the structure of the protocol, we employ channel simulation [37-39] and protocol stretching [38], originally devised for quantum communications. As depicted in Fig. 1(b), we consider an approximation $\mathcal{E}^{M}$ of the finite-dimensional channel $\mathcal{E}$ by applying a universal (trace-preserving) teleportation operation $\mathcal{T}^{M}$ to $M \geq 1$ copies of the Choi matrix $\rho_{\mathcal{E}}=(\mathcal{E} \otimes \mathcal{I}) \zeta$, where $\zeta:=\sum_{\ell=0}^{d-1}|\ell, \ell\rangle / \sqrt{d}$ is a maximally entangled state of dimension $d$. In general, $\mathcal{T}^{M}$ can be chosen as port-based teleportation (PBT) [40]. The precision of channel simulation is quantified by $\Delta_{\mathcal{E}, M}:=\left\|\mathcal{E}-\mathcal{E}^{M}\right\|_{\diamond}$ where $\|A\|_{\diamond}=\sup _{\rho}\|A \otimes \mathcal{I}(\rho)\|$ is the diamond norm $[5,41]$. For the simulation of an arbitrary finite-dimensional channel via PBT, we may write [[21] Lemma 2]

$$
\Delta_{\mathcal{E}, M} \leq \delta_{M, d}:=2 d(d-1) M^{-1},
$$

which is valid for any number of ports $M \geq 1$ and any input dimension $d \geq 2$ for the channel [42].

The error in the channel simulation propagates to the output of the protocol. Using the triangle inequality, we can bound the trace distance between the output state $\rho_{\mathcal{E}, u}$ of the actual protocol and the output state $\tilde{\rho}_{\mathcal{E}, u}$ of the simulated protocol as follows

$$
\left\|\rho_{\mathcal{E}, u}-\tilde{\rho}_{\mathcal{E}, u}\right\| \leq u \Delta_{\mathcal{E}, M} .
$$

The final step is protocol stretching [21,38]. As depicted in Fig. 1(c), this is a reorganization of the simulated protocol into an equivalent block protocol, so that the approximate output state $\tilde{\rho}_{\mathcal{E}, u}$ is decomposed as $\tilde{\rho}_{\mathcal{E}, u}=\Lambda\left(\rho_{\mathcal{E}}^{\otimes u M}\right)$ for a trace-preserving quantum operation $\Lambda$. Combining this with Eq. (4) we then write

$$
\left\|\rho_{\mathcal{E}, u}-\Lambda\left(\rho_{\mathcal{E}}^{\otimes u M}\right)\right\| \leq u \Delta_{\mathcal{E}, M} .
$$

Ultimate bounds. - Combining Lemma 1 with Eq. (5), we derive the main result of our work (proof in [33]).
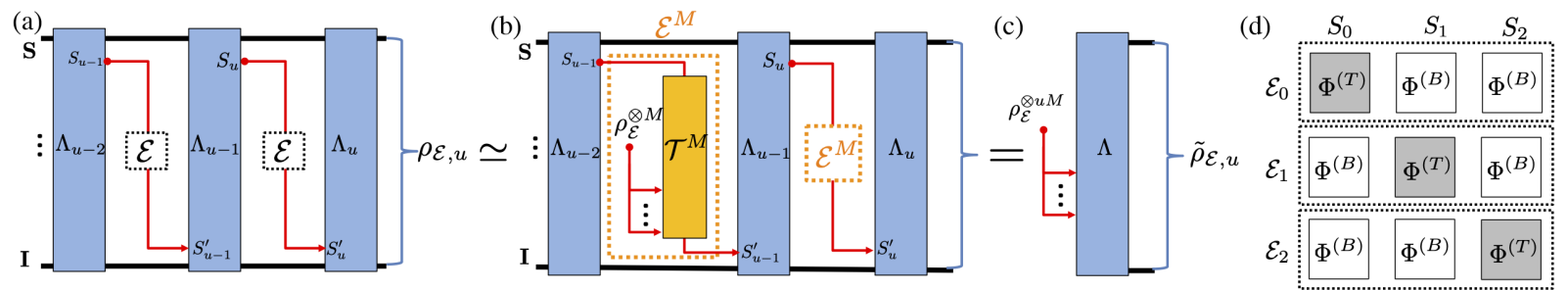

FIG. 1. Schematics of (a) a general adaptive protocol. The inputs $\mathbf{S}$ and $\mathbf{I}$ are quantum registers in an arbitrary state. In the $(k-1)$ th round, a subsystem $S_{k-1}$ probes the channel $\mathcal{E}$. A quantum operation $\Lambda_{k-1}$ is performed to process the received subsystem $S_{k-1}^{\prime}$ and prepare the next probe subsystem $S_{k}$. After $u$ uses, the final decision is made based on the measurement of the output state $\rho_{\mathcal{E}, u}$. (b) Channel simulation. A general protocol over channel $\mathcal{E}$ is replaced by a protocol over an approximate channel $\mathcal{E}^{M}$, consisting of a teleportation operation $\mathcal{T}^{M}$ applied to $M$ copies of the Choi matrix $\rho_{\mathcal{E}}$. (c) Protocol stretching. Starting from the simulated protocol in (b), all the $u$ copies of the resource state $\rho_{\mathcal{E}}^{\otimes M}$ are stretched back in time and all the quantum operations (together with the registers $\mathbf{S}$ and I) are collapsed into a single trace-preserving quantum operation $\Lambda$ that produces $\tilde{\rho}_{\mathcal{E}, u}$. (d) Channel position finding with $m=3$ multichannels $\mathcal{E}_{0}, \mathcal{E}_{1}, \mathcal{E}_{2}$, each acting on three subsystems $S_{0}, S_{1}, S_{2}$. Here $\Phi^{(T)}$ and $\Phi^{(B)}$ represent target and background channels, respectively (see also Ref. [24]). 
Theorem 1.-Consider arbitrary $m \geq 2 d$-dimensional quantum channels $\left\{\mathcal{E}_{n}\right\}_{n=0}^{m-1}$ with prior probabilities $\left\{p_{n}\right\}_{n=0}^{m-1}$. The minimum error probability $P_{u}$ for their $u$-round adaptive discrimination satisfies

$$
P_{u} \geq P_{u, L B}:=P_{H}\left(\left\{\rho_{\mathcal{E}_{n}}^{\otimes u M}, p_{n}\right\}\right)-u \bar{\Delta}_{M} / 2,
$$

where the average simulation error $\bar{\Delta}_{M}=\sum_{n} p_{n} \Delta_{\mathcal{E}_{n}, M}$ can be replaced by the uniform error $\delta_{M, d}$ of Eq. (3).

Since the bound is valid for any $M \geq 1$, its tightest value is achieved by maximizing over $M$. Remarkably, the difficult problem of adaptive multi-channel discrimination has been reduced to the discrimination of an ensemble of Choi matrices. However, in general, the computation of the Helstrom limit $P_{H}\left(\left\{\rho_{\mathcal{E}_{n}}^{\otimes u M}, p_{n}\right\}\right)$ may still be challenging and, for this reason, we may resort to further bounds. In particular, by using bounds from Bures's fidelity $F(\rho, \sigma):=\operatorname{tr} \sqrt{\sqrt{\rho} \sigma \sqrt{\rho}}$, we can obtain a lower bound that is easier to evaluate [33]

$$
P_{u} \geq P_{u, L B}^{F}=\sum_{k^{\prime}>k} p_{k^{\prime}} p_{k} F^{2 u M}\left(\rho_{\mathcal{E}_{k^{\prime}}}, \rho_{\mathcal{E}_{k}}\right)-u \bar{\Delta}_{M} / 2 .
$$

Below, we consider symmetric cases where the bound of Theorem 1 can be greatly simplified.

Ensembles with symmetries.-The general problem of adaptive multichannel discrimination can be further simplified if the ensemble possesses certain symmetries. The first to consider is joint telecovariance. A quantum channel $\mathcal{E}$ is telecovariant [38] when, for any teleportation unitary $U$ (e.g., Pauli operator), we may write $\mathcal{E}\left(U \rho U^{\dagger}\right)=V \mathcal{E}(\rho) V^{\dagger}$ for another generally different unitary $V$ (See [43-45] for general covariance.). Then, an ensemble of channels $\left\{\mathcal{E}_{k}\right\}$ is called jointly telecovariant $[9,22]$, when we may write the condition of telecovariance for all the elements of the ensemble and the output unitary $V$ does not depend on the label $k$, i.e., it is universal for the ensemble.

For an ensemble of jointly telecovariant channels, we may rewrite the previous universal simulation by choosing $\mathcal{T}^{M}$ as the standard teleportation [46] applied to a single Choi matrix $(M=1)$. Furthermore, this simulation is perfect, meaning that we have $\Delta_{\mathcal{E}, 1}=0$ [38]. As a result, Theorem 1 reduces to $P_{u, L B}=P_{H}\left(\left\{\rho_{\mathcal{E}_{n}}^{\otimes u}, p_{n}\right\}\right)$. Furthermore, this lower bound is achievable $\left(P_{u}=P_{u, L B}\right)$ by probing the channels with $u$ copies of the maximally entangled state $\zeta$, which also means that adaptive strategies are not needed for these channels. We have therefore automatically proved the following, which is a generalization of Ref. [[22] Theorem 3] from binary to multiary channel discrimination.

Corollary 1.-Consider arbitrary $m \geq 2$ jointly telecovariant channels $\left\{\mathcal{E}_{n}\right\}_{n=0}^{m-1}$ with prior probabilities $\left\{p_{n}\right\}_{n=0}^{m-1}$. The minimum error probability for their $u$-round adaptive discrimination equals the Helstrom limit computed over their Choi matrices

$$
P_{u}=P_{H}\left(\left\{\rho_{\mathcal{E}_{n}}^{\otimes u}, p_{n}\right\}\right) .
$$

This is achievable by a nonadaptive entanglement-based strategy where $u$ copies of a maximally entangled state $\zeta$ are sent through the extended channel $\mathcal{E}_{n} \otimes \mathcal{I}$.

Examples of jointly telecovariant channels are QECs and all Pauli channels, therefore including QDCs. By contrast, QADCs do not belong to this family.

We can perform another relevant simplification when the ensemble possesses GUS [23]; i.e., it has equal priors $p_{n}=1 / m$ and the channels satisfy $\mathcal{E}_{n}=S^{n} \mathcal{E}_{0} S^{\dagger n}$, where the unitary $S^{m}$ equals identity. In this case, the Choi matrices $\rho_{\mathcal{E}_{n}}^{\otimes u M}$ also have GUS with extended symmetry operators $S_{u M}=S^{\otimes u M}$. Then the optimal POVM $\left\{\Pi_{n}\right\}_{n=0}^{m-1}$ for discriminating a GUS ensemble of states has the same type of symmetry, i.e., $\Pi_{n}=S_{u M}^{n} \Pi_{0} S_{u M}^{\dagger n}[23,47]$. As a result, the lower bound in Theorem 1 takes the form

$$
P_{u, L B}=1-\frac{1}{2} u \Delta_{\mathcal{E}_{0}, M}-\max _{\Pi_{0}} \operatorname{Tr}\left[\Pi_{0} \rho_{\mathcal{E}_{0}}^{\otimes u M}\right],
$$

where the maximization is constrained by POVM normalization condition. Finally, if the channel ensemble has both the properties of GUS and joint telecovariance, then we may write the ultimate achievable bound

$$
P_{u}=1-\max _{\Pi_{0}} \operatorname{Tr}\left[\Pi_{0} \rho_{\mathcal{E}_{0}}^{\otimes u}\right]
$$

In the following, we consider $\mathrm{CPF}$, which has the property of GUS as a natural symmetry.

Channel position finding.-An important case where we have GUS is the problem of CPF [see Fig. 1(d) for a schematic]. Consider an array of $m$ cells, each containing a channel acting on a $d_{S}$-dimensional subsystem $S_{k}$. The goal is to find the position $n$ of a target channel $\Phi^{(T)}$, knowing that all the other cells contain copies of a background channel $\Phi^{(B)}$. Formally, we consider equal-prior discrimination of $m$ multichannels $\left\{\mathcal{E}_{n}\right\}_{n=0}^{m-1}$, each expressed by

$$
\mathcal{E}_{n}=\left(\otimes_{k \neq n} \Phi_{S_{k}}^{(B)}\right) \otimes \Phi_{S_{n}}^{(T)} .
$$

By taking $m$ maximally entangled states at the input $\zeta^{\otimes m}$, we define the global Choi matrix of the multichannel above, which has the following form

$$
\rho_{\mathcal{E}_{n}}=\left[\otimes_{k \neq n}\left(\rho_{\Phi^{(B)}}\right)_{S_{k} I_{k}}\right] \otimes\left(\rho_{\Phi^{(T)}}\right)_{S_{n} I_{n}} .
$$

From the multichannel $\mathcal{E}_{n}$ we can derive an $M$-port PBT simulation $\mathcal{E}_{n}^{M}$ by replacing each individual channel $\Phi^{(B / T)}$ with its $M$-port simulation. Correspondingly, the simulation error affecting the multichannel is in terms of the errors associated to the simulation of the individual channels, i.e., $\Delta_{\mathcal{E}_{n}, M}=(m-1) \Delta_{\Phi^{(B)}, M}+\Delta_{\Phi^{(T)}, M}$ [33]. Because this expression is the same for any $n$, the average simulation error is simply $\bar{\Delta}_{M}=\sum_{n} p_{n} \Delta_{\mathcal{E}_{n}, M}=\Delta_{\mathcal{E}_{0}, M}$. 
Furthermore, from Eq. (3) we have $\Delta_{\Phi^{(\ell)}, M} \leq \delta_{M, d_{S}}$, and we can write the simpler upper bound $\bar{\Delta}_{M} \leq$ $m \delta_{M, d_{S}} \sim m d_{S}^{2} / M$. The simulation error of the CPF problem can be used in previous equations. In particular, we can use it in Eq. (7), which here takes the form

$$
P_{u} \geq P_{u, L B}^{F}=\frac{m-1}{2 m} F_{\Phi^{(B)}, \Phi^{(T)}}^{4 u M}-u \bar{\Delta}_{M} / 2,
$$

where $F_{\Phi^{(B)}, \Phi^{(T)}}$ is the fidelity between the Choi matrices of the target and background channels [48].

In order to show further applications of our theory, below we consider three families of channels: QECs, QDCs, and QADCs. The first two are jointly telecovariant, so that our Corollary 1 and Eq. (10) can be applied.

Discrimination of erasure and depolarizing channels.Let us study the multiary discrimination of QECs and QDCs. Recall that the $d$-dimensional QEC with erasure probability $q$ can be written as $\mathcal{E}_{q}(\rho)=q|e\rangle\langle e|+(1-q) \rho$, where $\rho$ is the input state and $|e\rangle\langle e|$ is a state living in an orthogonal space. The $d$-dimensional QDC with depolarizing probability $q$ takes instead the form $\mathcal{D}_{q}(\rho)=q \mathbb{I}_{d}+(1-q) \rho$, where $\mathbb{I}_{d}=d^{-1} I$ is the fully mixed state. These two types of channels can be treated compactly by exploiting the formalism of the orthogonal replacement channel. This is explained in detail in [33], where we also show that, for the special case of binary discrimination between QECs (or QDCs), we find exact analytical solutions for the ultimate error probability.

Consider the multiary discrimination problem of CPF specified in Eq. (11). Here the background channel $\Phi^{(B)}$ and the target channel $\Phi^{(T)}$ are chosen to be QECs (or QDCs) with probabilities $q_{B}$ and $q_{T}$. For $m$ channels and $u$ uses, we define the function

$$
\begin{aligned}
h_{m}^{u}\left(q_{B}, q_{T}\right):= & 1-\frac{1}{m} \sum_{x \in\{0,1\}^{u m}}\left[q_{T}^{w^{\star}}\left(1-q_{T}\right)^{u-w^{\star}}\right. \\
& \left.\times q_{B}^{\|\boldsymbol{x}\|-w^{\star}}\left(1-q_{B}\right)^{(m-1) u-\left(\|x\|-w^{\star}\right)}\right],
\end{aligned}
$$

where $w^{\star}=\max _{\ell}\left\|\boldsymbol{x}_{\ell}\right\|$ for $q_{T} \geq q_{B}$, while $w^{\star}=\min _{\ell}\left\|\boldsymbol{x}_{\ell}\right\|$ [49] for $q_{T}<q_{B}$. Here $\boldsymbol{x}_{\ell}$ (with $0 \leq \ell \leq m-1$ ) is the $(1+\ell u)$ th to $[(\ell+1) u]$ th components of the vector $\boldsymbol{x}$. Note that $h_{m}^{u}\left(q_{B}, q_{T}\right)=h_{m}^{u}\left(1-q_{B}, 1-q_{T}\right)$. Using this function, we compute $P_{u}$ in Eq. (10) and, when $u=1$, the summation can be simplified analytically [33].

For CPF with QECs $\Phi^{(B)}=\mathcal{E}_{q_{B}}$ and $\Phi^{(T)}=\mathcal{E}_{q_{T}}$, we find the ultimate error probability

$$
P_{u}^{\mathrm{QEC}}=h_{m}^{u}\left(q_{B}, q_{T}\right) .
$$

In this case there is no entanglement advantage, since we obtain the same performance by sending $u$ copies of an optimal pure state $\phi^{\otimes m}$ through $\mathcal{E}_{n}$ in a nonadaptive fashion. For CPF with QDCs $\Phi^{(B)}=\mathcal{D}_{q_{B}}$ and $\Phi^{(T)}=\mathcal{D}_{q_{T}}$, we compute the ultimate error probability

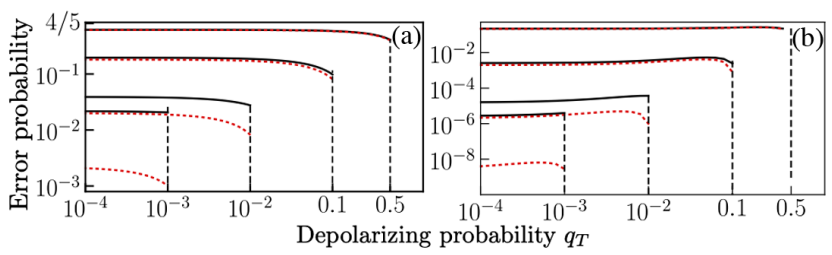

FIG. 2. Channel position finding with QDCs $\Phi^{(B)}=\mathcal{D}_{q_{B}}$ and $\Phi^{(T)}=\mathcal{D}_{q_{T}}$. We consider $m=5, d=100$, and $q_{B}-q_{T}=0.5$, $0.9,0.99,0.999$ from top to bottom. We compare the ultimate (entanglement-based) performance $P_{u}^{\mathrm{QDC}}$ of Eq. (16) (red curves) with the optimal classical strategy based on unentangled inputs (black curves). (a) $u=1$, (b) $u=3$. In all panels, the vertical dashed lines are the maximum values that $q_{T}$ can take, because for those values we have $q_{B}=1$.

$$
P_{u}^{\mathrm{QDC}}=h_{m}^{u}\left[\left(1-d^{-2}\right) q_{T},\left(1-d^{-2}\right) q_{B}\right] .
$$

In this case, there is instead a clear advantage in using entanglement, since the performance of an optimal pure state $\phi^{\otimes m}$ is given by Eq. (16) with the replacement $d^{-2} \rightarrow d^{-1}$ [33]. Figure 2 shows the gap between the entangled and nonentangled strategy which widens as the difference $\left|q_{B}-q_{T}\right|$ increases, and as the number of rounds $u$ increases. For one-shot discrimination $(u=1)$ of a completely depolarizing channel $q_{T}=1$ among identity channels $\left(q_{B}=0\right)$, we may write $P_{1}^{\mathrm{QDC}}=$ $(m-1) / m d^{2}[33]$.

Discrimination of amplitude damping channels.-A QADC $\mathcal{A}_{q}$ with damping probability $q$ has Kraus decomposition $\mathcal{A}_{q}(\rho)=\sum_{i=0,1} K_{i} \rho K_{i}^{\dagger}$, with operators $K_{0}:=$ $|0\rangle\langle 0|+\sqrt{1-q}| 1\rangle\langle 1|$ and $K_{1}:=\sqrt{q}|0\rangle\langle 1|$. It is not telecovariant and its PBT simulation has nonzero error $\Delta_{\mathcal{A}_{q}, M}=\xi_{M}[(1-q) / 2+\sqrt{1-q}]$, where $\xi_{M}$ is the constant given in Ref. [[21] Eq. (11)]. While the binary discrimination between two QADCs has been treated in the literature [21] (see [33] for further results on receiver designs and pretty-good measurement (PGM) [25-27]), little is known in the setting of multiary discrimination.

Consider the multi-ary discrimination problem of CPF specified in Eq. (11), with background $\Phi^{(B)}=\mathcal{A}_{q_{B}}$ and target $\Phi^{(T)}=\mathcal{A}_{q_{T}}$. We compute the lower bound in Eq. (13) here taking the form

$$
P_{u} \geq P_{u, L B}^{F}=\frac{m-1}{2 m} F^{4 u M}-u \bar{\Delta}_{M} / 2,
$$

where $F:=\left[1+\sqrt{\left(1-q_{B}\right)\left(1-q_{T}\right)}+\sqrt{q_{B} q_{T}}\right] / 2$ and $\bar{\Delta}_{M}=(m-1) \Delta_{\mathcal{A}_{q_{B}}, M}+\Delta_{\mathcal{A}_{q_{T}}, M}$. By optimizing over $M$, we derive its tightest form $P_{u, L B}^{F \star}=\max _{M} P_{u, L B}^{F}$. As a comparison, we consider a nonadaptive scheme, where $u$ copies of the maximally entangled state $\zeta^{\otimes m}$ probe $\mathcal{E}_{n}$. Correspondingly, the Helstrom limit computed on the ensemble of output Choi matrices $\left\{\rho_{\mathcal{E}_{n}}^{\otimes u}\right\}$ is bounded as [33] 


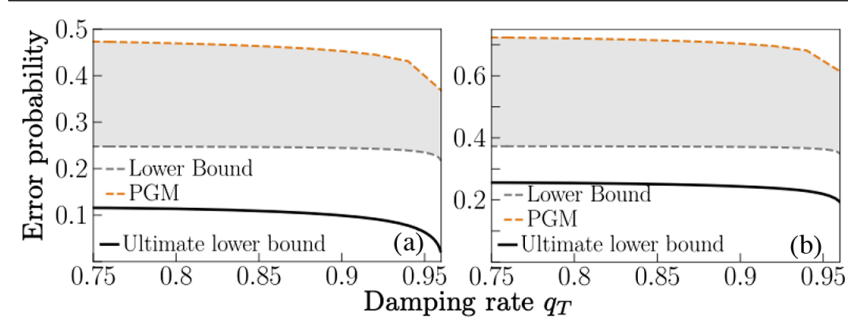

FIG. 3. Channel position finding with QADCs $\mathcal{A}_{q_{B}}$ and $\mathcal{A}_{q_{T}}$ for $q_{B}=q_{T}+0.04$. The solid black curve is the ultimate lower bound $P_{u, L B}^{F \star}$ optimized from Eq. (17). The nonadaptive performance lies between the fidelity lower bound (gray dashed curve) and the PGM upper bound (orange dashed curve) as given by Eq. (18). (a) We consider $m=2$ and $u=4$. (b) We consider $m=4$ and $u=2$.

$$
\frac{m-1}{2 m} F^{4 u} \leq P_{H}\left(\left\{\rho_{\mathcal{E}_{n}}^{\otimes u}, 1 / m\right\}\right) \leq P_{E}^{\mathrm{PGM}},
$$

where $P_{E}^{\mathrm{PGM}}$ is the performance achievable via a PGM at the output [25-27]. Figure 3 shows a gap between the ultimate lower bound $P_{u, L B}^{F \star}$ and the nonadaptive performance. Further investigation is needed to establish if this gap is effectively due to adaptiveness.

Conclusions.-In this work, we established the ultimate limits for the minimum error probability affecting the (generally adaptive) statistical discrimination of an arbitrary $m \geq 2$ number of finite-dimensional quantum channels. We find remarkable simplifications in the presence of symmetries, with our bound becoming exactly achievable when the channel ensemble is jointly telecovariant. Our theory allows us to find the ultimate performances achievable in the fundamental $m$-ary discrimination problem of $\mathrm{CPF}$, considering various types of channels. In particular, for CPF with depolarizing channels, we show that the use of entanglement greatly outperforms the performance of any classical strategy.

Note that CPF can be translated into various applications, including readout of memories, radar scanning and absorbance spectroscopy. For instance, CPF may model the readout process from a digital memory where information is encoded in the position of a target cell within a block. In the frequency domain, this is equivalent to finding the absorbance line within a spectrum. A possible future direction is developing our theory in the setting of unambiguous hypothesis testing, suitably extending Refs. [4,50-53] to $m$-ary channel discrimination.

Q. Z. acknowledges funding from Army Research Office under Grant No. W911NF-19-1-0418, Office of Naval Research under Grant No. N00014-19-1-2189, Defense Advanced Research Projects Agency (DARPA) under Young Faculty Award (YFA) Grant No. N660012014029, and University of Arizona. S. P. acknowledges funding from the European Union's Horizon 2020 Research and Innovation Action under
Grant Agreement No. 862644 (Quantum readout techniques and technologies, QUARTET).

*zhuangquntao@email.arizona.edu

[1] C. Helstrom, Quantum Detection and Estimation Theory, Mathematics in Science and Engineering: A Series of Monographs and Textbooks (Academic Press, New York, 1976).

[2] A. Chefles and S. M. Barnett, Quantum state separation, unambiguous discrimination and exact cloning, J. Phys. A 31, 10097 (1998).

[3] A. Chefles, Quantum state discrimination, Contemp. Phys. 41, 401 (2000).

[4] J. A. Bergou, Discrimination of quantum states, J. Mod. Opt. 57, 160 (2010).

[5] A. Y. Kitaev, Quantum computations: Algorithms and error correction, Russ. Math. Surv. 52, 1191 (1997).

[6] A. Acín, E. Jané, and G. Vidal, Optimal estimation of quantum dynamics, Phys. Rev. A 64, 050302(R) (2001).

[7] M. F. Sacchi, Entanglement can enhance the distinguishability of entanglement-breaking channels, Phys. Rev. A 72, 014305 (2005).

[8] G. Wang and M. Ying, Unambiguous discrimination among quantum operations, Phys. Rev. A 73, 042301 (2006).

[9] S. Pirandola, B. R. Bardhan, T. Gehring, C. Weedbrook, and S. Lloyd, Advances in photonic quantum sensing, Nat. Photonics 12, 724 (2018).

[10] S.-H. Tan, B. I. Erkmen, V. Giovannetti, S. Guha, S. Lloyd, L. Maccone, S. Pirandola, and J. H. Shapiro, Quantum Illumination with Gaussian States, Phys. Rev. Lett. 101, 253601 (2008).

[11] Q. Zhuang, Z. Zhang, and J. H. Shapiro, Optimum MixedState Discrimination for Noisy Entanglement-Enhanced Sensing, Phys. Rev. Lett. 118, 040801 (2017).

[12] Q. Zhuang, Z. Zhang, and J. H. Shapiro, Entanglementenhanced Neyman-Pearson target detection using quantum illumination, J. Opt. Soc. Am. B 34, 1567 (2017).

[13] Q. Zhuang, Z. Zhang, and J.H. Shapiro, Quantum illumination for enhanced detection of Rayleigh-fading targets, Phys. Rev. A 96, 020302(R) (2017).

[14] Z. Zhang, S. Mouradian, F. N. C. Wong, and J. H. Shapiro, Entanglement-Enhanced Sensing in a Lossy and Noisy Environment, Phys. Rev. Lett. 114, 110506 (2015).

[15] S. Pirandola, Quantum Reading of a Classical Digital Memory, Phys. Rev. Lett. 106, 090504 (2011).

[16] R. Takagi, B. Regula, K. Bu, Z.-W. Liu, and G. Adesso, Operational Advantage of Quantum Resources in Subchannel Discrimination, Phys. Rev. Lett. 122, 140402 (2019).

[17] A. W. Harrow, A. Hassidim, D. W. Leung, and J. Watrous, Adaptive versus nonadaptive strategies for quantum channel discrimination, Phys. Rev. A 81, 032339 (2010).

[18] A. Acin, Statistical Distinguishability between Unitary Operations, Phys. Rev. Lett. 87, 177901 (2001).

[19] R. Duan, Y. Feng, and M. Ying, Perfect Distinguishability of Quantum Operations, Phys. Rev. Lett. 103, 210501 (2009).

[20] R. Duan, Y. Feng, and M. Ying, Entanglement is Not Necessary for Perfect Discrimination between Unitary Operations, Phys. Rev. Lett. 98, 100503 (2007). 
[21] S. Pirandola, R. Laurenza, C. Lupo, and J. L. Pereira, Fundamental limits to quantum channel discrimination, npj Quantum Inf. 5, 50 (2019).

[22] S. Pirandola and C. Lupo, Ultimate Precision of Adaptive Noise Estimation, Phys. Rev. Lett. 118, 100502 (2017).

[23] G. Cariolaro and G. Pierobon, Theory of quantum pulse position modulation and related numerical problems, IEEE Trans. Commun. 58, 1213 (2010).

[24] Q. Zhuang and S. Pirandola, Entanglement-enhanced testing of multiple quantum hypotheses, Commun. Phys. 3, 103 (2020).

[25] A. S. Holevo, On asymptotically optimal hypotheses testing in quantum statistics, Teor. Veroyatnost. i Primenen 23, 429 (1978) [Theory Probab. Appl. 23, 411 (1979)].

[26] P. Hausladen and W. K. Wootters, A 'pretty good' measurement for distinguishing quantum states, J. Mod. Opt. 41, 2385 (1994).

[27] P. Hausladen, R. Jozsa, B. Schumacher, M. Westmoreland, and W. K. Wootters, Classical information capacity of a quantum channel, Phys. Rev. A 54, 1869 (1996).

[28] H. Barnum and E. Knill, Reversing quantum dynamics with near-optimal quantum and classical fidelity, J. Math. Phys. (N.Y.) 43, 2097 (2002).

[29] E. Bagan, J. A. Bergou, S. S. Cottrell, and M. Hillery, Relations between Coherence and Path Information, Phys. Rev. Lett. 116, 160406 (2016).

[30] D. Qiu and L. Li, Minimum-error discrimination of quantum states: Bounds and comparisons, Phys. Rev. A 81, 042329 (2010).

[31] T. Ogawa and H. Nagaoka, Strong converse to the quantum channel coding theorem, IEEE Trans. Inf. Theory 45, 2486 (1999).

[32] A. Montanaro, A lower bound on the probability of error in quantum state discrimination, in 2008 IEEE Information Theory Workshop (IEEE, New York, 2008), pp. 378-380.

[33] See the Supplemental Material at http://link.aps.org/ supplemental/10.1103/PhysRevLett.125.080505 for details of proofs and calculations, which includes Refs. [34-36].

[34] C. A. Fuchs and J. van de Graaf, Cryptographic distinguishability measures for quantum mechanical states, IEEE Trans. Inf. Theory 45, 1216 (1999).

[35] H. Yuen, R. Kennedy, and M. Lax, Optimum testing of multiple hypotheses in quantum detection theory, IEEE Trans. Inf. Theory 21, 125 (1975).

[36] Y. C. Eldar, A. Megretski, and G. C. Verghese, Optimal detection of symmetric mixed quantum states, IEEE Trans. Inf. Theory 50, 1198 (2004).

[37] M. A. Nielsen and I. L. Chuang, Programmable Quantum Gate Arrays, Phys. Rev. Lett. 79, 321 (1997).
[38] S. Pirandola, R. Laurenza, C. Ottaviani, and L. Banchi, Fundamental limits of repeaterless quantum communications, Nat. Commun. 8, 15043 (2017).

[39] L. Banchi, J. Pereira, S. Lloyd, and S. Pirandola, Convex optimization of programmable quantum computers, npj Quantum Inf. 6, 42 (2020).

[40] S. Ishizaka and T. Hiroshima, Asymptotic Teleportation Scheme as a Universal Programmable Quantum Processor, Phys. Rev. Lett. 101, 240501 (2008).

[41] V. I. Paulsen, Completely Bounded Maps and Operator Algebras (Cambridge University Press, Cambridge, England, 2002).

[42] In the following, where we refer to a $d$-dimensional channel, we refer to the dimension of the input Hilbert space. The dimension of the output Hilbert space could be different, as it happens, for instance, for an erasure channel.

[43] A. S. Holevo, Remarks on the classical capacity of quantum channel, arXiv:quant-ph/0212025.

[44] N. Datta, M. Fukuda, and A. S. Holevo, Complementarity and additivity for covariant channels, Quantum Inf. Process. 5, 179 (2006).

[45] Q. Zhuang, E. Y. Zhu, and P. W. Shor, Additive Classical Capacity of Quantum Channels Assisted by Noisy Entanglement, Phys. Rev. Lett. 118, 200503 (2017).

[46] C. H. Bennett and S. J. Wiesner, Communication Via One-and Two-Particle Operators on Einstein-PodolskyRosen States, Phys. Rev. Lett. 69, 2881 (1992).

[47] N. Dalla Pozza and G. Pierobon, Optimality of square-root measurements in quantum state discrimination, Phys. Rev. A 91, 042334 (2015).

[48] This comes from Eq. (7) where we use the expression of the multichannel Choi matrix in Eq. (12), the multiplicativity of the fidelity under tensor products, and the fact that the priors are equal, so that $\sum_{k^{\prime}>k} p_{k^{\prime}} p_{k} \rightarrow[(m-1) / 2 m]$. The expression of the simulation error can also be exploited in Eq. (9) which explicitly accounts for the GUS property of the CPF problem.

[49] Here $|\boldsymbol{x}|=\sum_{i}\left|\boldsymbol{x}_{i}\right|$ is the vector one norm.

[50] Y. Sun, J. A. Bergou, and M. Hillery, Optimum unambiguous discrimination between subsets of nonorthogonal quantum states, Phys. Rev. A 66, 032315 (2002).

[51] A. Chefles, Condition for unambiguous state discrimination using local operations and classical communication, Phys. Rev. A 69, 050307(R) (2004).

[52] U. Herzog and J.A. Bergou, Optimum unambiguous discrimination of two mixed quantum states, Phys. Rev. A 71, 050301 (2005).

[53] M. Kleinmann, H. Kampermann, and D. Bruß, Unambiguous discrimination of mixed quantum states: Optimal solution and case study, Phys. Rev. A 81, 020304(R) (2010). 